\title{
LATTICE-INVARIANT TOPOLOGICAL PROPERTIES
}

\author{
P. L. SHARMA
}

\begin{abstract}
The purpose of this paper is to show that an isomorphism between the lattices of all closed sets of two topological spaces induces an isomorphism on the lattices of their zero-sets. This is achieved by showing that any continuous real-valued function on a space $X$ can be transferred to any space latticeequivalent to $X$. Several topological properties are shown to be lattice-invariant.
\end{abstract}

1. Introduction. The concept of "homeomorphism" of two topological spaces is generalized by requiring the lattices of their open sets to be isomorphic. The idea was perhaps introduced by H. J. Kowalsky [6] who gave a long list of lattice-invariant topological properties which include complete regularity, normality, complete normality and paracompactness, among others. Later W. J. Thron [2] showed that two $T_{D^{-}}$-spaces are lattice equivalent iff they are homeomorphic.

In this paper we show that an isomorphism between the lattices of closed sets of two spaces induces an isomorphism between the lattices of their zero-sets. This is done by showing that continuous real-valued functions from a topological space $X$ can be "transferred" to any space latticeequivalent to $X$. Then, it easily follows that realcompactness, pseudocompactness, paracompactness and some of its weaker forms, extremal disconnectedness, basic disconnectedness, perfect normality, etc. are lattice-invariant topological properties.

2. Terminology and basic facts. The interior and the closure of a set $A$ in a topological space are denoted by $A^{\circ}$ and $\bar{A}$ (or $\left.\operatorname{cl}(A)\right)$ respectively. $C(X)$ is the set of all continuous real-valued functions on $X$. The collection of all zero-sets of $X$ is $Z(X)$, and $Z(f)$ denotes the zero-set of a real-valued function $f$. Two subsets $A$ and $B$ of a topological space $X$ are said to be normally separable iff there exists a continuous function $f: X \rightarrow[0,1]$ such that $f(A)=0$ and $f(B)=1$. The set of all natural numbers starting with 1 is denoted by $N$.

Received by the editors September 13, 1971.

AMS 1970 subject classifications. Primary 54A10, 54C30; Secondary 54C50.

Key words and phrases. Lattice-equivalent topological spaces, lattice-invariant property, zero sets.

(c) American Mathematical Society 1972 
Recall that two topological spaces are lattice-equivalent iff their lattices of open sets (or equivalently closed sets) are isomorphic. A topological property $P$ is said to be lattice-invariant if a topological space latticeequivalent to a topological space having $P$ has also $p$. Throughout we shall assume that the partial ordering under consideration is the set inclusion.

Let $X$ and $Y$ be two lattice-equivalent topological spaces. If $\lambda_{1}$ is an isomorphism from the lattice $\tau$ of open sets of $X$ onto the lattice $\tau^{\prime}$ of open sets of $Y$ then, letting $\zeta$ and $\zeta^{\prime}$ be the lattices of closed subsets of $X$ and $Y$ respectively, we denote by $\lambda_{2}$ the isomorphism between the lattices $\zeta$ and $\zeta^{\prime}$ defined by $\lambda_{2}(F)=Y-\lambda_{1}(X-F)$ for each $F$ in $\zeta$. Now we define a one-one function $\lambda_{12}$ from $\tau \cup \zeta=\{A \subset X: A \in \tau$ or $A \in \zeta\}$ onto $\tau^{\prime} \cup \zeta^{\prime}=\{B \subset Y$ : $B \in \tau^{\prime}$ or $\left.B \in \zeta^{\prime}\right\}$ by setting $\lambda_{12}(A)=\lambda_{1}(A)$ or $\lambda_{2}(A)$ according as $A \in \tau$ or $A \in \zeta$. Note that $\lambda_{1}(A \cup B)=\lambda_{1}(A \vee B)=\lambda_{1}(A) \vee \lambda_{1}(B)=\lambda_{1}(A) \cup \lambda_{1}(B)$ and similarly for $\cap$ and $\wedge$. The same is true for $\lambda_{2}$ and any two members $A, B$ of $\zeta$. Thus if $A$ is a clopen subset of $X$ then $\lambda_{2}(A)=Y-\lambda_{1}(X-A)$ and since $\lambda_{1}$ is an isomorphism, $\lambda_{1}(A)$ and $\lambda_{1}(X-A)$ are disjoint with their union being equal to $Y$. This shows that $\lambda_{1}(A)=\lambda_{2}(A)$ and thus $\lambda_{12}$ is well defined. The fact that $\lambda_{12}$ is one-one and onto is obvious. We list some properties of $\lambda_{12}$ in the following lemma.

Lemma. The function $\lambda_{12}: \tau \cup \zeta \rightarrow \tau^{\prime} \cup \zeta^{\prime}$ has the following properties:

(a) $\lambda_{12}$ preserves order; i.e. given two members $A, B$ of $\tau \cup \zeta$ with $A \subset B$, then $\lambda_{12}(A) \subset \lambda_{12}(B)$.

(b) $\lambda_{12}\left(A^{\circ}\right)=\left(\lambda_{12}(A)\right)^{\circ}$ for each $A \in \tau \cup \zeta$.

(c) $\lambda_{12}(\bar{A})=\operatorname{cl}\left(\lambda_{12}(A)\right)$ for each $A \in \tau \cup \zeta$.

Proof. (a) If $A$ and $B$ are both open or both closed then we are through. If $A$ is open and $B$ is closed then $A$ and $X-B$ are disjoint open subsets of $X$ and so $\lambda_{1}(A)$ and $\lambda_{1}(X-B)$ are disjoint open subsets of $Y$. Therefore $\lambda_{1}(A) \subset Y-\lambda_{1}(X-B)=\lambda_{2}(B)$, i.e. $\lambda_{12}(A) \subset \lambda_{12}(B)$. A similar argument can be given when $A$ is closed and $B$ is open.

(b) The existence of an open subset $Q$ of $Y$ which properly contains $\lambda_{12}\left(A^{\circ}\right)$ and is contained in $\lambda_{12}(A)$ implies the existence of an open subset $P$ of $X$ which properly contains $A^{\circ}$ and is contained in $A$ (by (a)).

The proof of (c) is similar.

3. Some results. Roughly speaking our next two theorems state that if a topological space $X$ has a rich supply of continuous real-valued functions then any space $Y$ lattice-equivalent to $X$ must also have "enough" continuous real-valued functions.

THeORem 3.1. Let $(X, \tau)$ and $\left(Y, \tau^{\prime}\right)$ be two lattice-equivalent topological spaces with an isomorphism $\lambda_{1}$ from $\tau$ onto $\tau^{\prime}$. Suppose $f \in C(X)$ and $f \geqq 0$. 
Then there exists $f^{*} \in C(Y)$ having the following properties:

(a) $\lambda_{12}(Z(f))=Z\left(f^{*}\right)$.

(b) $f^{*}$ is bounded iff $f$ is bounded.

Proof. For a real number $t>0$, let $G_{t}=\{x \in X: f(x)<t\}$ and let $\lambda_{12}\left(G_{t}\right)=F_{t}$. By Lemma 2.1, $\lambda_{12}\left(\operatorname{cl}\left(G_{t}\right)\right)=\bar{F}_{t}$. For $y \in Y$, let $f^{*}(y)=$ $\inf \left\{t: y \in F_{t}\right\}$. Then for any positive real number $r, f^{*}(y)<r$ iff $y$ is in $F_{t}$ for some $t<r$; and $f^{*}(y)>r$ iff $y$ is not a member of at least one $\bar{F}_{t}$ for some $t>r$. This proves that $f^{*}$ is continuous. It is obvious that $f^{*}$ is bounded iff $f$ is bounded. Also $Z(f)=\bigcap\left\{\bar{G}_{t}: t>0\right\}$ and $Z\left(f^{*}\right)=\bigcap\left\{\bar{F}_{t}: t>0\right\}$ showing thereby that $\lambda_{12}(Z(f))=Z\left(f^{*}\right)$. Hence the theorem is proved.

The restriction of the mapping $\lambda_{12}$ to $Z(X)$ in the above theorem is an isomorphism between the lattices $Z(X)$ and $Z(Y)$. This, in turn, induces an order-preserving one-one mapping from the set of all $Z$-filters on $X$ onto the set of all $Z$-filters on $Y$; the maximal (prime) $Z$-filters on $X$ correspond to the maximal (resp. prime) $Z$-filters on $Y$. We summarise these facts in the following theorem.

THEOREM 3.2. Any isomorphism between the lattices of closed sets of two lattice-equivalent spaces $X$ and $Y$ establishes an isomorphism between the lattices of their zero-sets. Also there exists an order-preserving one-one correspondence between the sets of Z-filters of such spaces.

From the above theorem and the fact that there exists a one-one mapping from the set of all $Z$-ultrafilters of a space $X$ onto the set of all maximal ideals of $C(X)$, we get the following result.

COROLlary. If two topological spaces $X$ and $Y$ are lattice-equivalent then the sets of the maximal ideals of $C(X)$ and $C(Y)$ are in a one-one correspondence.

Recall that (i) a topological space is completely regular iff the zero-sets form a base for the closed sets, and (ii) two sets in a topological space are normally-separable iff they are contained in disjoint zero-sets. Using these facts and Theorem 3.2 we get the following results, which are due to Kowalsky [6] and were later proved by Wong [3] and Thron [2].

COROLlary. Complete regularity is lattice-invariant.

COROLlaRY. Normality is lattice-invariant.

Let $X$ be a topological space and $\tau$ be the lattice of all open sets. Then $X$ is paracompact iff for any family $\beta \subset \tau$ such that $\bigvee\{B: B \in \beta\}=X$, there corresponds a family $\zeta \subset \tau$ satisfying the following three conditions:

(i) $\bigvee\{C: C \in \zeta\}=X$.

(ii) For each $C \in \zeta$ there exists $B \in \beta$ such that $C<B$.

(iii) There exists $\mu \subset \tau$ such that $\bigvee\{M: M \in \mu\}=X$, and for any $M \in \mu, M \wedge C=0$ for all but a "finitely many" $C \in \zeta$. 
To characterise para-Lindelöf property, we have only to replace the phrase "finitely many" by "countably many" in (iii) above.

Using Lemma 2.1, Theorems 3.1 and 3.2, and such lattice characterizations of topological properties as above, we have

THEOREM 3.3. Realcompactness, pseudocompactness, paracompactness, countable paracompactness, para-Lindelöf, extremal disconnectedness, basic disconnectedness and countable compactness are lattice-invariant properties.

Proof. Let $X$ and $Y$ be two lattice-equivalent spaces.

Suppose $X$ is realcompact and let $\mathscr{F}^{*}$ be a $Z$-ultrafilter on $Y$ with the countable intersection property. Then $\mathscr{F}=\left\{F \in \zeta: \lambda_{2}(F) \in \mathscr{F} *\right\}$ is a $Z$ ultrafilter on $X$ with the countable intersection property. Then the intersection $P$ of all members of $\mathscr{F}$ is a nonempty closed subset of $X$. Obviously the nonempty set $\lambda_{2}(P)$ is the intersection of all members of $\mathscr{F}^{*}$. This shows that $Y$ is realcompact.

If $X$ is not pseudocompact then there exists an unbounded continuous real-valued function $f$ on $X$ such that $f(x) \geqq 0$ for each $x \in X$. Let $t$ be any positive number. Then $G_{t}=\{x \in X: f(x)<t\}$ is a proper open subset of $X$ and therefore $F_{t}=\lambda_{12}\left(G_{t}\right)$ is a proper open subset of $Y$. For any $y \in Y-F_{t}$, $f^{*}(y) \geqq t$ and so $f^{*}$ is unbounded. Thus $Y$ is not pseudocompact.

The remaining parts of this theorem can be proved easily. The case of paracompactness was settled earlier by Kowalsky [6].

THeOREM 3.4. Perfect normality is lattice-invariant.

Proof. Let two topological spaces $X$ and $Y$ be lattice-equivalent and let $Y$ be perfectly normal. So $X$ is normal. Take a closed subset $A$ of $X$ and let $\lambda_{12}(A)=B$. Then there exists a decreasing sequence $\left(H_{n}\right), n \in N$, of open subsets of $Y$ such that $\bigcap\left\{H_{n}: n \in N\right\}=B$. Let $\lambda_{12}\left(G_{n}\right)=H_{n}$. By Lemma 2.1, $A \subset G_{n}$ and so for each $n \in N$ we can find an open set $P_{n}$ such that $A \subset$ $P_{n} \subset \bar{P}_{n} \subset G_{n} ;$ which implies that $A \subset \bigcap\left\{P_{n}: n \in N\right\} \subset \bigcap\left\{\bar{P}_{n}: n \in N\right\} \subset$ $\bigcap\left\{G_{n}: n \in N\right\}$. Applying Lemma 2.1 again, we get $A=\bigcap\left\{P_{n}: n \in N\right\}$. Hence the theorem is proved.

The following example shows that metacompactness is not a latticeinvariant property.

EXAMPLE. Let $X$ be the set of all nonzero integers and let $Y=X \cup\{0\}$. For a positive integer $n$, let $P_{n}=\{k \in X: k \geqq n\}, P_{-n}=P_{n} \cup\{-n\}, Q_{n}=$ $P_{n} \cup\{0\}$ and $Q_{-n}=P_{-n} \cup\{0\}$. Let $\tau$ be the topology on $X$ for which the collection $\left\{P_{n}: n\right.$ is a nonzero integer $\}$ is a base.

Similarly let $\tau^{\prime}$ be the topology on $Y$ generated by the base $\left\{Q_{n}: n\right.$ is a nonzero integer $\}$. Then $(X, \tau)$ and $\left(Y, \tau^{\prime}\right)$ are lattice-equivalent, $Y$ is not metacompact but $X$ is. 
The lattice invariance of compactness, local compactness, second countability, etc. was proved by Yim-Ming Wong [3]. He has also given an example to show that first countability is not lattice-invariant. But the example given by Wong [3] to show that separability and complete normality are not lattice-invariant 'seems erroneous; his claim that the two topological spaces constructed in the example are lattice-equivalent appears incorrect. In fact the lattice-invariance of complete normality was already established by Kowalsky [6]. To show that separability is not latticeinvariant, we refer to the last example in Wong's paper [3].

Professor S. A. Naimpally is due special thanks for his encouragement and advice. The referee is due additional thanks for bringing $H$. J. Kowalsky's paper [6] to the author's notice and also for other helpful suggestions.

\section{REFERENCES}

1. G. Birkoff, Lattice theory, rev. ed., Amer. Math. Soc. Colloq. Publ., vol. 25. Amer. Math. Soc., Providence, R.I., 1948. MR 10, 673.

2. W. J. Thron, Lattice-equivalence of topological spaces, Duke Math. J. 29 (1962), 671-679. MR 26 \#4307.

3. Yim-Ming Wong, Lattice invariant properties of topological spaces, Proc. Amer. Math. Soc. 26 (1970), 206-208.

4. L. Gillman and M. Jerison, Rings of continuous functions, University Series in Higher Math., Van Nostrand, Princeton, N.J., 1960. MR 22 \#6994.

5. J. L. Kelley, General topology, Van Nostrand, Princeton, N.J., 1955. MR 16, 1136.

6. H. J. Kowalsky, Verbandstheoretische Kennzeichnung topologischer Räume, Math. Nachr. 21 (1960), 297-318. MR 22 \#9952.

Department of Mathematics, Indian Institute of Technology, Kanpur-16, INDIA 